\title{
Magnetic Levitation Device for Harvesting Power-frequency Magnetic Field and Mechanical Energy
}

\author{
Wei HE* \\ School of Information Engineering, Baise University, \\ Baise 533000, China \\ e-mail: weiheky@yeah.net \\ Chi-wen QU \\ School of Information Engineering, Baise University, \\ Baise 533000, China \\ Gao-feng CHE \\ School of Information Engineering, Baise University, \\ Baise 533000, China
}

\author{
Yue-ran LU \\ School of Information Engineering, Baise University, \\ Baise 533000, China
}

\author{
Cheng-de LIN \\ School of Information Engineering, Baise University, \\ Baise 533000, China
}

Ji-tao ZHANG

College of Electric and Information Engineering, Zhengzhou University of Light Industry, Zhengzhou 450002, China

\begin{abstract}
Energy harvesting from ambient energy sources has been receiving considerable interest as the power supply of wireless sensors networks. In this paper, a hybrid energy harvester based on magnetic levitation is presented for scavenging power-frequency magnetic field and mechanical energy. A Halbach array is employed to enhance the power output. At the magnetic field of $15 \mathrm{Oe}$ and the vibration acceleration of $80 \mathrm{mg}$, the harvester produces a load power of $0.3 \times 10^{-6} \mathrm{~W}$ across a matching load resistance of $4.4 \mathrm{M} \Omega$. The device has the potential applications in energy harvesting for low-power electronic devices.
\end{abstract}

Keywords-Harvester; Magnetic levitation; Power-frequency magnetic field; Vibration.

\section{INTRODUCTION}

The power supply of wireless sensors has impeded the development and application of wireless sensors networks due to the limited lifetime of the batteries. The advances of electronic technology decrease the power consumption of wireless sensors, and scavenging energy from external environments has become an alternative of the traditional batteries. [1-5]. There are many energy sources for power generation [6-8], and the mechanisms harvesting two or more energy can attain a higher energy conversion efficiency $[9,10]$.

Power-frequency magnetic field and vibration are promising energy sources due to their ubiquitous. Energy harvesters based on magnetoelectric (ME) transducers have attracted much attention due to the high magnetomechanical coupling effect [11-13], and they can simultaneously scavenge magnetic field and vibration energy.

This paper presents a hybrid energy harvester for Power-frequency magnetic field and vibration. The magnetic levitation unit attains a low spring rate and the Halbach array achieves a particular magnetic field distribution. The experiment results have validated the proposed design.

\section{ENERGY HARVESTING DEVICE}

Fig. 1 shows the schematic diagram of the proposed hybrid energy harvester. The harvester consists of a magnetic levitation unit, a Halbach array, and a ME transducer. The Halbach array concentrates the magnetic field of the array on the side where the ME tranducer is placed, which can potentially improve the power output of the device. The ME transducer is composed of a piezoelectric PZT5H layer sandwiched between two magnetostrictive Terfenol-D layers. The magnetostrictive layers are magnetized in length direction and the piezoelectric layer is polarized along thickness direction.

Under power-frequency magnetic field and vibrational excitations (or one of the two excitations), the magnetostrictive layers of the ME tranducer are deformed. The stress is transmitted to the piezoelectric layer. Consequently, the piezoelectric layer produce power output on the connected load resistance due to the piezoelectric effect [14].

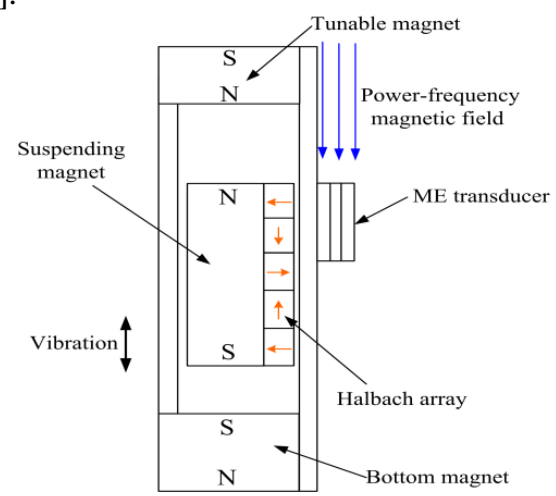

Figure 1. Schematic diagram of the hybrid energy harvester. 


\section{MAGNETOELECTRIC ANALYSIS}

Based on the principle of the ME transducer, when the external magnetic fields are exerted on the ME transducer, the open-circuit output voltage can be expressed as [15]

$$
V_{0}=\alpha_{\mathrm{ME}} \times\left(\Delta H+H_{a c}\right)
$$

where $\alpha_{M E}$ is the magnetoelectric coefficient, $\Delta H$ is the magnetic field variation caused by the vibration, and $\mathrm{H}_{\mathrm{ac}}$ is the external power-frequency magnetic field. When a resistor is connected to the ME transducer, the maximum output power can be calculated by

$$
P_{\max }=\frac{V_{o p t}^{2}}{R_{o p t}}=\omega C_{p} V_{o p t}^{2}
$$

where $\mathrm{V}_{\mathrm{opt}}$ is the voltage on the optimal external load resistance $R_{o p t}, \omega$ is the angular frequency, and $C_{p}$ is the equivalent capacitance of the ME transducer.

\section{RESULTS AND DISCUSSIONS}

Fig. 2 shows the experimental results of the harvester under the magnetic field excitation of $\mathrm{H}_{\mathrm{ac}}=10$ Oe near 50 Hz. It can be seen from Fig. 2 that the harvester has a relatively flat response as the frequency is increased from 40 $\mathrm{Hz}$ to $60 \mathrm{~Hz}$, indicating a non-resonant state. The average output voltage reaches $0.5826 \mathrm{~V}$ for the given data of Fig. 2 .

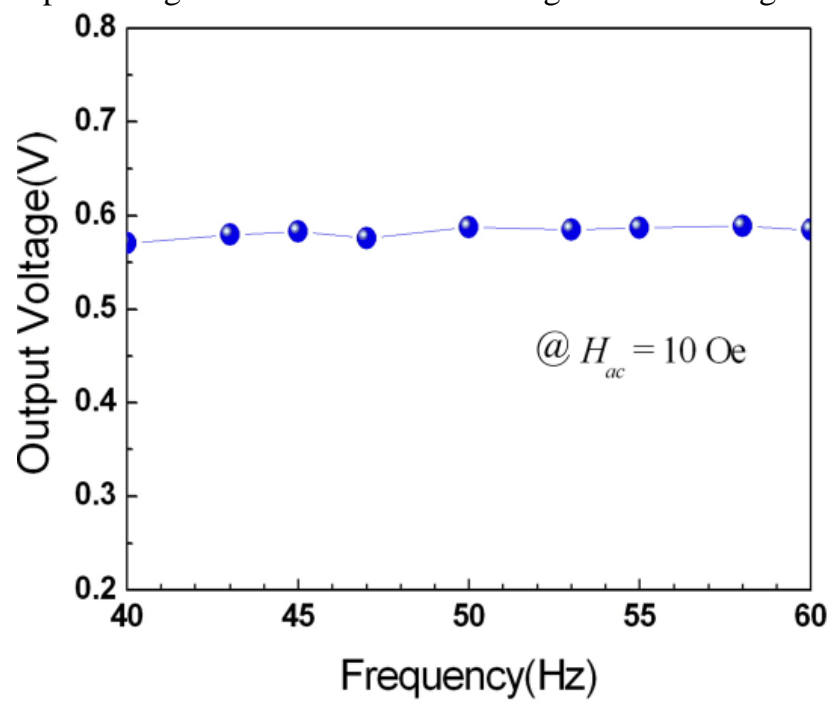

Figure 2. Experimental response of the device under only magnetic field excitation around $50 \mathrm{~Hz}$.

The harvester is exposed to both power-frequency magnetic field and vibrational excitations. The magnetic field and the vibration acceleration are 15 Oe and $80 \mathrm{mg}$, respectively. A resistance box is connected to the hybrid energy harvester to test power output performance. The results are plotted in Fig. 3. As can be seen from Fig. 3 that, the output power increases with the load resistance, and attains the maximum value of $0.3 \times 10^{-6} \mathrm{~W}$ with the load resistance of $4.4 \mathrm{M} \Omega$. Then, the power decreases with further increase of the load resistance.

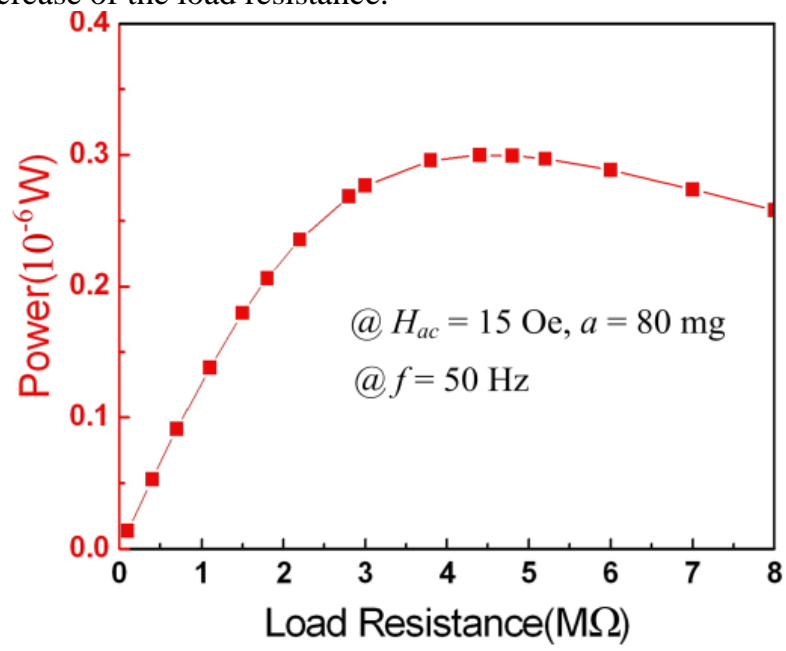

Figure 3. Output power as a function of load resistance under both magnetic field and vibrational excitations at $50 \mathrm{~Hz}$.

\section{CONCLUSIONS}

In this paper, an energy harvester based on magnetic levitation is presented. The harvester can simultaneously scavenge external power-frequency magnetic field and vibration energy. A Halbach array is used to increase the magnetic field variation which the ME transducer undergoes. Experiments have been carried out. The results show that the harvester can generate a load power of $0.3 \times 10^{-6} \mathrm{~W}$ on a 4.4 $\mathrm{M} \Omega$ matching resistance under dual excitations. The power output can be significantly increased by increasing the external excitations and using a ME transducer with higher ME voltage coefficient (e.g., Terfenol - D/Pb $\left(\mathrm{Mg}_{1 / 3} \mathrm{Nb}_{2 / 3}\right) \mathrm{O}_{3}$ $-\mathrm{PbTiO}_{3} /$ Terfenol-D), which indicates the potential applications of the device for wireless sensors.

\section{ACKNOWLEDGMENT}

This work is supported by the National Natural Science Foundation of China (Grant Nos. 61540039 and 61503344) and the Natural Science Foundation of Guangxi Province (Grant No. 2015GXNSFBA139263).

\section{REFERENCES}

[1] A. A. Nasir, X. Zhou, S. Durrani, and R. A. Kennedy, Relaying protocols for wireless energy harvesting and information processing, IEEE Trans. Wireless Commun. 12, 3622 (2013).

[2] S. Adhikari, M. I. Friswell, and D. J. Inman, Piezoelectric energy harvesting from broadband random vibrations, Smart Mater. Struct 18, 115005 (2009).

[3] W. He, P. Li, Y. Wen, J. Zhang, A. Yang, and C. Lu, A noncontact magnetoelectric generator for energy harvesting from power lines, IEEE Trans. Magn. 50, 8204604 (2014).

[4] D. Zhu, S. Beeby, J. Tudor, and N. Harris, Vibration energy harvesting using the Halbach array, Smart Mater. Struct. 21, 075020 (2012).

[5] W. He, P. Li, Y. M. Wen, J. T. Zhang, A. C. Yang, C. J. Lu, J. Yang, J. Wen, Y. Zhu, J. Qiu, and M. Yu, Piezoelectric energy harvester scavenging AC magnetic field energy from electric power lines, Sens. Actuators A 193, 59 (2013). 
[6] H. Wang, J. D. Park, Z. J. Ren, Practical energy harvesting for microbial fuel cells: a review, Environ. Sci. Technol. 49, 3267 (2015).

[7] C. P. Le, and E. Halvorsen, MEMS electrostatic energy harvesters with end-stop effects, J. Micromech. Microeng. 22, (074013) 2012.

[8] N. Wu, X. Cheng, Q. Zhong, J. Zhong, W. Li, B. Wang, and J. Zhou, Cellular polypropylene piezoelectret for human body energy harvesting and health monitoring, Adv. Func. Mater. 25, 4788 (2015).

[9] S. X. Dong, J. Y. Zhai, J. F. Li, D. Viehland, and S. Priya, Multimodal system for harvesting magnetic and mechanical energy, Appl. Phys. Lett. 93, 103511(2008).

[10] A. Bibo, M. F. Daqaq, Investigation of concurrent energy harvesting from ambient vibrations and wind using a single piezoelectric generator, Appl. Phys. Lett. 102, 243904 (2013).

[11] X. Z. Dai, Y. M. Wen, P. Li, J. Yang, and G. Y. Zhang, Modeling, characterization and fabrication of vibration energy harvester using
Terfenol-D/PZT/Terfenol-D composite transducer, Sens. Actuators A 156, 350 (2009)

[12] W. He, P. Li, Y. Wen, J. Zhang, A. Yang, and C. Lu, Energy harvesting from two-wire power cords using magnetoelectric transduction, IEEE Trans. Magn. 50, 4004805 (2014).

[13] Y. Zhu, and J. W. Zu, A magnetoelectric generator for energy harvesting from the vibration of magnetic levitation, IEEE Trans. Magn. 48, 3344 (2012).

[14] A. Arnau, Piezoelectric transducers and applications, 2 ed. (Heidelberg, Germany: Springer, 2008).

[15] S. X. Dong, J. F. Li, and D. Viehland, Longitudinal and transverse magnetoelectricvoltage coefficients of magnetostrictive/piezoelectric laminate composite: theory, IEEE Trans. Ultrason. Fekroelectr. Freq. Control 50, 1253 (2003) 